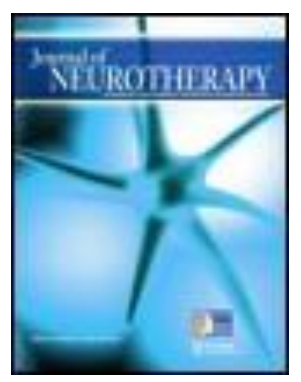

Journal of Neurotherapy: Investigations in Neuromodulation, Neurofeedback and Applied Neuroscience

\title{
International Society for Neuronal Regulation European Chapter (ISNR-EU) Inaugural Conference
}

David L. Trudeau MD , Donald Bars PhD , Marco Congedo PhD , David Joffe BA, Joel Lubar PhD,

Tobias Egner PhD , Thomas F. Zech PhD , John H. Gruzelier PhD , Sebern Fischer MA,

V. A. Grin-latsenko PhD , Yu. D. Kropotov PhD , V. A. Ponomarev PhD , L. S. Chutko PhD , E.

A. Yakovenko, John Gruzelier PhD , Tobias Egner PhD, Wolfgang Klimesch PhD, Roberto D. Pascual-Marqui PhD , Michaela Esslen PhD , Dietrich Lehmann MD , Dr. Jordan Pop-Jordanov , Dr. Nada Pop-Jordanova MD , Dr. Natasa Markovska , Beverly Steffert PhD , M. Barry Sterman PhD , Tanju Surmeli MD , Michael Thompson MD , Lynda Thompson PhD, David Vernon PhD, Tobias Egner PhD, Nick Cooper MSc, Theresa Compton BSc, Claire Neilands BSc , Amna Sheri BSc , John Gruzelier PhD , Edwin Verstraeten PhD \& Raymond Cluydts PhD

Published online: 08 Sep 2008.

To cite this article: David L. Trudeau MD, Donald Bars PhD , Marco Congedo PhD, David Joffe BA, Joel Lubar PhD, Tobias Egner PhD , Thomas F. Zech PhD , John H. Gruzelier PhD, Sebern Fischer MA, V. A. Grin-latsenko PhD , Yu. D. Kropotov PhD , V. A. Ponomarev PhD , L. S. Chutko PhD , E. A. Yakovenko, John Gruzelier PhD , Tobias Egner PhD , Wolfgang Klimesch PhD , Roberto D. Pascual-Marqui PhD, Michaela Esslen PhD, Dietrich Lehmann MD , Dr. Jordan Pop-Jordanov , Dr. Nada PopJordanova MD , Dr. Natasa Markovska , Beverly Steffert PhD , M. Barry Sterman PhD , Tanju Surmeli MD , Michael Thompson MD , Lynda Thompson PhD , David Vernon PhD , Tobias Egner PhD , Nick Cooper MSc, Theresa Compton BSc, Claire Neilands BSc , Amna Sheri BSc , John Gruzelier PhD , Edwin Verstraeten PhD \& Raymond Cluydts PhD (2004) INTERNATIONAL SOCIETY FOR NEURONAL REGULATION EUROPEAN CHAPTER (ISNR-EU) INAUGURAL CONFERENCE, Journal of Neurotherapy: Investigations in Neuromodulation, Neurofeedback and Applied Neuroscience, 8:1, 83-102, DOI: $\underline{10.1300 / J 184 \mathrm{~V} 08 \mathrm{n} 01 \_08}$

To link to this article: http://dx.doi.org/10.1300/J184v08n01_08

\section{PLEASE SCROLL DOWN FOR ARTICLE}

(C) International Society for Neurofeedback and Research (ISNR), all rights reserved. This article (the "Article") may be accessed online from ISNR at no charge. The Article may be viewed online, stored in electronic or physical form, or archived for research, teaching, and private study purposes. The Article may be archived in public libraries or university libraries at the direction of said public library or university library. Any other reproduction of the Article for redistribution, sale, resale, loan, sublicensing, systematic supply, or other distribution, including both physical and electronic reproduction for such purposes, is expressly forbidden. Preparing or reproducing derivative works of this article is expressly forbidden. ISNR makes no representation or warranty as to the accuracy or completeness of any content in the Article. From 1995 to 2013 the Journal of Neurotherapy was the official publication of ISNR (www. Isnr.org); on April 27, 2016 ISNR acquired the journal from Taylor \& Francis Group, LLC. In 2014, ISNR established its official open-access journal NeuroRegulation (ISSN: 2373-0587; www.neuroregulation.org).

THIS OPEN-ACCESS CONTENT MADE POSSIBLE BY THESE GENEROUS SPONSORS

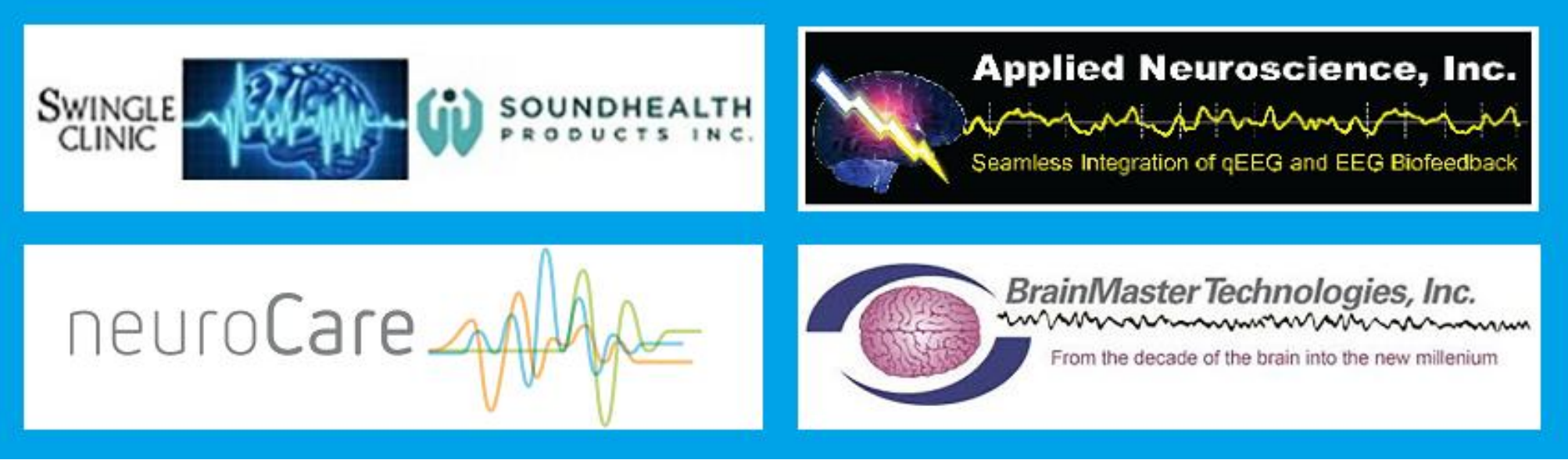




\begin{tabular}{c}
\hline INTERNATIONAL SOCIETY \\
FOR NEURONAL REGULATION \\
EUROPEAN CHAPTER (ISNR-EU) \\
INAUGURAL CONFERENCE \\
\hline
\end{tabular}

\section{Submitted Abstracts of Papers}

\section{EDITORIAL NOTE: \\ INTRODUCTION TO SCIENTIFIC ABSTRACTS, ISNR-EU 2003}

The most recent and exciting scientific information is often presented at conferences long before it makes its way through the peer review process into print. As a compromise between timeliness and thorough review, we have chosen to print these abstracts of scientific papers presented at the inaugural meeting of the ISNR-EU chapter. This meeting was held conjointly with the Biofeedback Foundation of Europe (BFE) in Udine, Italy during February 2003. The empiric papers were reviewed by the ISNR-EU conference program committee. The discussion papers were not so reviewed.

David L. Trudeau, MD

Editor

Journal of Neurotherapy, Vol. 8(1) 2004

Copyright (C) 2004 ISNR. All rights reserved.

Digital Object Identifier: 10.1300/J184v08n01_08 


\section{ABSTRACTS}

\section{The Addition of Sensory Evoked Potentials to qEEG Data to Im- prove Clinical Outcomes}

\section{Donald Bars, PhD}

Research Consultant, University of Basel, Medical School, Psychiatric and Neurological Clinics

$<$ the.bars@balcab.ch>

The use of sensory evoked responses (evoked potentials [EP]), can improve the effectiveness of therapeutic interventions. Evoked potential information is different than the data from other imaging techniques in that it registers responses that occur within the first three- to fourtenths of a second post stimuli, most of which is an obligatory response to sensory reception. This decreases effects of other electrophysiological variables entering the results as more cognitive networks and environmental factors become involved in processing the presented stimuli.

This presentation addressed several clinical research studies as the basis for gaining a beginning understanding of relationships between specific visual and auditory EPs and behaviours. A basic background will allow an understanding of what an EP is, how an EP is created, and important considerations for clinical use. Several different paradigms were presented and discussed, including how the created waveforms have been associated with a variety of brain functioning. Research was presented concerning separation of explosive, out-of-control behaviours into several different physiological groups, a physiologically type of ruminating behaviour and when combined with qEEG results can separate affective disorders and disorders of attention.

\section{Electromagnetic Tomographic Neurofeedback: A Pilot Study}

Marco Congedo, PhD (1,2), David Joffe, BA (3), and Joel Lubar, PhD (1)

(1) Brain Research and Neurophysiology Laboratory, Department of Psychology, The University of Tennessee, Knoxville, (2) Nova Tech EEG, Inc., Knoxville, Tennessee, (3) Lexicor Medical Technology, Inc., Boulder, Colorado

$<$ loretabiofeedback@yahoo.com>

Journal of Neurotherapy, Vol. 8(1) 2004

Copyright (C) 2004 ISNR. All rights reserved.

Digital Object Identifier: 10.1300/J184v08n01_09 


\section{Background}

A major limitation of current neurofeedback paradigma is the limited information provided by a single or a small number of electrodes placed on the scalp. A considerable improvement of the neurofeedback efficacy and specificity could be obtained feeding back brain activity of delimited structures. While traditional EEG information reflects the superposition of the electrical activity of a large number of neurons, by means of inverse solutions such as the Low-Resolution Electromagnetic Tomography (LORETA) spatially delimited brain activity can be evaluated in neocortical tissue.

\section{Methods}

Three individuals were trained to improve brain activation (suppress low alpha [8-10 Hz] and enhance beta [16-20 Hz] current density) in the anterior cingulate gyrus cognitive division (ACcd). Participants took part in six experimental sessions, each lasting approximately 30 minutes. Six EEG baselines were recorded for each subject before the training started. Permutation tests were conducted on baseline recordings before and after the beginning of the training. In addition a randomized trial was performed at the end of the treatment. During eight two-minutes periods (trials) participants were asked to try to obtain as many rewards as they could (4 "plus" trials) or as few rewards as they could (4 "minus" trials). The order of trials was decided at random. The hypothesis under testing was that participants acquired volitional control over their brain activity so to be able to obtain more rewards during the plus condition as compared to the minus condition.

\section{Results}

An exact test of the randomized trials showed evidence of volitional control for two subjects $(\mathrm{p}=0.043$ and $\mathrm{p}=0.1)$ and no evidence of volitional control for one of them $(\mathrm{p}=0.271)$. The combination of the three p-values provided an overall probability value for this experiment of 0.012 with the additive method and 0.035 with the multiplicative method. These results support the hypothesis of volitional control across the experimental group. Pre- and post-treatment brain changes are currently under analysis. 


\section{Conclusions}

With as few as six training sessions, typically insufficient to produce any form of learning with scalp neurofeedback, the experiment showed overall signs of volitional control of the electrical activity of the ACcd. While the pilot study intended to test technical issues, we are now carrying out a larger study to test the ability to change intracranial electrical activity. Tomographic neurofeedback has never been done before. Possible applications of the technique are important and include the treatment of epileptic foci, the treatment of specific brain regions damaged as a consequence of traumatic brain injury, and in general, of any specific cortical electrical activity.

\section{The Effects of Neurofeedback Training on Spectral Topography of the Healthy Electroencephalogram \\ Tobias Egner, PhD (1), Thomas F. Zech, PhD (2), and John H. Gruzelier, $\operatorname{PhD}(3)$ \\ (1) Functional Research Center, Columbia University, (2) Department of Clinical Psychology and Psychotherapy, Philips University, Marburg, Germany, (3) Department of Cognitive Neuroscience \& Behaviour, Fac- ulty of Medicine, Imperial College, London \\ $<$ te2111@ columbia.edu>}

\section{Background}

Although important strides have been made towards establishing the clinical efficacy of neurofeedback of frequency components, a precise understanding of the impact of neurofeedback training on post-training spectral electroencephalographic (EEG) topography, which is presumed to mediate any cognitive-behavioural effects, remains elusive. In order to elucidate the influence of commonly applied neurofeedback protocols on spectral EEG composition, two studies involving healthy participants were carried out.

\section{Methods}

In the first experiment, 22 subjects were trained on SMR, beta1, and alpha/theta protocols, with resting and on-task spectral EEG measures assessed before and after training. The specific associations between 
learning indices of each individual training protocol and changes in delta, theta, alpha, SMR, beta1, and beta 2 absolute and relative activity levels in 11 scalp regions were assessed by means of partial correlation analyses. The results of experiment one served to generate hypotheses for experiment two, where 26 subjects were randomly allocated to independent groups of SMR, beta1, and alpha/theta training. Spectral resting and on-task EEG measures again were recorded prior and subsequent to training, and analysed for significant changes within each group.

\section{Results}

Few associations between particular protocols and spectral EEG changes were found to be consistent across the two studies, and these did not correspond to expectations based on the operant contingencies trained. SMR training was found to be associated with reduced SMR levels in the relative resting EEG, while alpha/theta training was reliably associated with increased general slow wave (delta/theta) and reduced frontal beta band (beta1/beta2) activity in the relative resting EEG.

\section{Conclusions}

The results documented that neurofeedback of frequency components does affect spectral EEG topography in healthy subjects, but that these effects do not necessarily correspond to either the frequencies or the scalp locations addressed by the training protocols, underlines the complexity of the neural dynamics involved. The association between alpha/theta training and replicable reductions in frontal beta activity can be interpreted as empirical neurophysiological evidence supporting the conceptualisation of this training regime as reducing agitation and anxiety.

\section{Fear and FPO2: The Implications of a New Protocol}

Sebern Fischer, MA

Private Practice, EEG Spectrum International, Northampton, Massachusetts

<sebern.fisher@verizon.net>

\section{Background}

Many problematic behaviors are driven by fear, though there is no direct mention of fear in the DSM-IV. Fear or fear-based memory is a 
common element in a number of psychopathological conditions including: Asperger's Syndrome, Post-Traumatic Stress Disorder, Generalized Anxiety Disorder, Reactive Attachment Disorder, Borderline Personality Disorder, Dissociative Identity Disorder, and many addictive disorders. The amygdala is responsible for generating fear. LeDoux (1996) and others postulate that the amygdala plays a central role in fear-based memory.

According to LeDoux, the low road from the amygdala leads directly to the orbito frontal area of the brain. This relationship suggests the hypothesis that employing neurofeedback to train the amygdala may provide a mechanism for addressing fear-associated disorders.

\section{Methods}

A site located below FP2 on the 10/20 system was chosen to allow optimal access to the orbital frontal afferent pathway to the amygdala. This site has been designated as FPO2. Twenty-six individuals were trained at this site in a clinical setting. The details of session data were discussed. Subjective reports were obtained following each session and logged in session notes.

\section{Results}

Patient's subjective reports included a reduction in fear, as well as a sense of calm and well-being, after training at FPO2. This change in affect appears to persist after the neurofeedback session.

\section{Discussion}

In addition to the 26 patients treated at the FPO2 site, a substantial body of anecdotal experience provided by other practitioners appears to confirm the efficacy of the protocol. Neurofeedback training at the FPO2 site provides a new protocol for clinically reducing fear level, as well as reducing the negative affect associated with fear-based memories.

\section{REFERENCE}

LeDoux, J. (1996). The emotional brain. New York: Simon \& Schuster. 


\section{Effect of Biofeedback Training on Sensorimotor and Beta 1 EEG} Rhythms on Attention Parameters

V. A. Grin-Iatsenko, PhD, Yu. D. Kropotov, PhD, V. A. Ponomarev, PhD, L. S. Chutko, PhD, and E. A. Yakovenko, Pre-Doctoral Fellow Institute of the Human Brain of Russian Academy of Sciences, St. Petersburg, Russia

\section{Introduction}

The aim of this study was to estimate the efficacy of bioregulation of the beta 1 rhythm and SMR in children with attention deficit diagnosed by complex examination including parent's questioning, results of psychological testing, and analysis of the components of cognitive evoked potentials.

\section{Methods}

The electrophysiological parameters of attention and action suppression were studied before and after a neurofeedback training course using recordings of cognitive evoked potentials (EP) during the performance of an auditory Go/NoGo test. Previous studies of cognitive evoked potentials in healthy children and teenagers showed that Go/NoGo tests caused a late positive EP deviation, localized in the frontal sites, and its amplitude was decreased in ADHD children.

The training protocol consisted of rewarding enhanced EEG amplitudes in the 12-18 Hz frequency band range, while simultaneously inhibiting excessive amplitudes in the low frequency $(1-7.5 \mathrm{~Hz})$ and high frequency $(19-30 \mathrm{~Hz})$ band range. The montage was bipolar with $\mathrm{C} 3-\mathrm{Fz}$ or $\mathrm{C} 4-\mathrm{Pz}$ in the standard 10-20 system. Left-side (C3) and right-side (C4) training involved rewarding activity in the $15-18 \mathrm{~Hz}$ and $12-15 \mathrm{~Hz}$, respectively. These two protocols were used in succession during a single training session with the respective duration (e.g., 20 minutes beta, 7-10 minutes SMR). Training consisted of 30 minutes of visual feedback. Each EEG biofeedback course consisted of 15 to 22 sessions (17 sessions on average) conducted two to five times per week for five to eight weeks.

\section{Results}

The dynamics of the components of cognitive evoked potentials in patients who successfully completed the training course showed a sig- 
nificant decrease in the amplitude of the N2 (after first and second stimuli) and an increase in the amplitude of the P3 (after second stimuli) components in the frontal and central sites.

\section{Conclusions}

A significant positive change in the amplitude of cognitive evoked potentials in the frontal and central areas in ADHD children trained by neurofeedback may point to the recovery of functioning of the action selection system and the mechanisms of attention.

\section{Investigating the Use of EEG Biofeedback in the Enhancement of} Music Performance

John Gruzelier, PhD (1) and Tobias Egner, PhD (2)

(1) Department of Cognitive Neuroscience \& Behaviour, Faculty of Medicine, Imperial College, London, (2) Functional Research Center, Columbia University

$<$ j.gruzelier@ic.ac.uk>

\section{Background}

Biofeedback-assisted modulation of electroencephalographic (EEG) activity (neurofeedback) has recently been shown to improve cognitive performance and affect event-related brain potentials in healthy humans (Egner \& Gruzelier, 2001). These data encouraged a series of investigations into the potential of neurofeedback for enhancing normal function on a non-laboratory measure: music performance in conservatoire students.

\section{Methods/Results}

In one study, participants were randomly allocated to either training on various neurofeedback protocols only, neurofeedback training plus physical and cognitive exercises, or a no-training control group. Music performances were video-recorded prior and subsequent to training, randomised, and evaluated by blind expert judges. Trends for improvement in musical performance were exclusive to the group that received neurofeedback training only, and improvements were highly correlated with learning to progressively raise theta $(5-8 \mathrm{~Hz})$ over alpha $(8-11 \mathrm{~Hz})$ band amplitudes (alpha/theta protocol). 
In a replication study participants were randomly allocated to groups of alpha/theta neurofeedback, SMR neurofeedback, beta1 neurofeedback, physical exercise, cognitive exercise, or Alexander technique training. The results replicated those of the first study, as only the alpha/theta training group displayed significant music performance improvement.

\section{Conclusions}

These data demonstrated the potential for slow wave neurofeedback to improve a complex set of skills in a real-life context.

\section{REFERENCE}

Egner, T. \& Gruzelier, J. H. (2001). Learned self-regulation of EEG frequency components affects attention and event-related brain potentials in humans. NeuroReport, 12 (18), 4155-4160.

Memory, Cognitive Performance and EEG Frequencies in the Theta and Alpha Range

Wolfgang Klimesch, PhD

Department of Physiological Psychology, University of Salzburg, Salzburg, Austria

<wolfgang.klimesch@mh.sbg.ac.at>

\section{Introduction}

Experiments from our laboratory suggest that event-related desynchronization (ERD) in the upper alpha band reflects semantic long-term memory processes; whereas, event-related synchronization (ERS) in the theta band reflects working memory processes. These findings were investigated with respect to interindividual differences in ERD/ERS, resting power and memory performance.

\section{Methods}

In a series of memory experiments spectral estimates for a resting period (resting power) and ERD/ERS during task performance were calculated. ERD/ERS is the percentage of a band power change during task 
performance with respect to a reference period preceding task performance.

\section{Results}

The findings show that memory performance-and cognitive performance in general-does not only depend on the magnitude of ERD/ERS but on resting power as well. We found that good performance is related to two types of EEG phenomena: (a) an increase in alpha but a decrease in theta resting power, and (b) a large alpha ERD but a large theta ERS, depending on the type of memory demands. Based on these findings we recently performed a repetitive transcranial magnetic stimulation (rTMS) study (in collaboration with Ch. Gerloff, University of Tübingen). The aim was to improve cognitive performance by applying rTMS at IAF during a reference interval before the performance of a mental rotation task on a trial-by-trial basis. We have found that rTMs at IAF (but not at $20 \mathrm{~Hz}$ and IAF-3 Hz) led to improved cognitive performance and increased ERD due to an increase in reference, but a decrease in task-related alpha power.

\section{Discussion}

The reported findings indicate that the relationship between alpha oscillations and cognitive performance is causal rather than correlative in nature. This has strong implications for biofeedback training because it is expected that an increase in individual alpha activity will increase cognitive performance.

\section{A New Method for the Computation of Cortical Connectivity with sLORETA (Standardized Low Resolution Brain Electromagnetic Tomography) \\ Roberto D. Pascual-Marqui, PhD, Michaela Esslen, PhD, and Dietrich Lehmann, MD \\ The KEY Institute for Brain-Mind Research, University Hospital of Psychiatry, Zurich, Switzerland <pascualm@key.unizh.ch>}

\section{Background}

A complex object such as the human brain is composed of a very large number of neurons, with a very large number of connections be- 
tween neurons. Classical functional imaging (PET and fMRI) has emphasized the localization of function, but has practically neglected the role played by functional connections. Oscillations and synchronization, which emerge because of cortical interconnections, have been hypothesized to play an essential role in cognition and consciousness. This work presents some novel methods for the computation and imaging of cortical connectivity.

\section{Methods}

Three-dimensional (3D) functional imaging of electric neuronal generators is performed with standardized low resolution brain electromagnetic tomography (sLORETA). This new method is uniquely capable of exact localization. In addition, it has the lowest spatial dispersion as compared to other published 3D linear, discrete, distributed EEG/MEG tomographies. Technical details and proof of properties for sLORETA are specified in Pascual-Marqui (2002). sLORETA provides high time resolution signals of "virtually implanted electrodes" throughout the cortex. Within any given time window, cortical connectivity is modelled in the following way: throughout the cortex, neuronal ensembles have the same dynamics of activation, except for location-specific scale factors and for location-specific latency shifts.

\section{Results}

In visual event-related potentials to stimulation with pictures of human faces, time lagged connections between primary/secondary visual cortices and the fusiform gyrus ("the face area") were demonstrated.

\section{Conclusions}

Validation was presented for methods that solve an important problem in the neurosciences: the imaging of specialized brain areas and their interconnections.

\section{On EEG Biofeedback Efficiency in Treating Cognitive Disorders} Jordan Pop-Jordanov, Prof. Dr. (1), Nada Pop-Jordanova, Prof. Dr. MD (2), and Natasa Markovska, Dr. (1)

(1) Macedonian Academy of Science and Arts, (2) Department of Psychophysiology, Paediatric Clinic, Faculty of Medicine, Skopje, Macedonia $<$ jpj@manu.edu.mk> 
EEG biofeedback is ultimately based on mental-neural information flow, but all standard attempts to explain the mechanism of the subtle two-way interaction between non-material mental agencies and neural events were confronted with violation of the energy-matter conservation laws of physics. In this presentation, the consecutive steps of biofeedback signal flow were investigated, considering the fundamental hypothesis of Eccles (Nobelist for Physiology and Medicine), which presumes that mental events cause neural events analogously to the probability fields of quantum mechanics. Applying an advanced quantum mechanical modelling, we calculated the probabilities for quantum transitions within neural molecules in cortical electric field. The results indicated the brain-wave frequency adjustment as an observable information-bearing physical mechanism. Therefore, some basic rationales for biofeedback efficiency in treating neurological disorders were deduced. In particular, the procedure for attention deficit mitigation through brain wave frequency adjustment (i.e., synchronization) was studied. As an example, the EEG biofeedback technique was applied to a group of ADD children resulting in an improvement of beta-theta ratio, as well as intelligence test scores and school grades.

\section{QEEG Analysis of Visual Dyslexics with Coloured Glasses Under Various Lighting Conditions}

Beverly Steffert, PhD

Birkbeck College, University of London

$<$ DrSteffert@aol.com>

\section{Background}

Dyslexia has generally been regarded as a linguistic deficit involving the segmentation of words into phonemes (speech sounds) despite early research identifying abnormal eye movements in $75 \%$ of dyslexics. However, no investigation of visual acuity had ever identified any cause until the concept of scotopic sensitivity became known in the 80s which identified cortical level deficits in the visual pathways. This paralleled known auditory sound discrimination deficits which cause difficulty in segmenting words at the visual level; the print appears to move and blur, letters can "jump" out of sequence and reading is effortful, slow and tiring. Coloured (spectrum-specific) lenses have previously benefited visual dyslexics. The present research has been funded by an optometric 
lens manufacturer to assess objectively any benefits to account for the subjective experience of benefit previously reported by coloured lens wearers.

\section{Methods}

Forty-three subjects ( 7 to 13 years old) were recruited from an advertisement detailing symptoms of Visual Dyslexia and screened by an optician. Subjects were given coloured lenses (without any optical correction) according to their improvement in pattern perception under a spectrum of coloured light conditions. These measurements were independent of optically corrected lenses.

QEEGs were recorded under five conditions (eyes closed, eyes open, reading in daylight, reading in daylight with their coloured lenses, reading in mixed fluorescent and daylight with their coloured lenses). A range of psychometric and balance/coordination tests were also given with and without coloured lenses. QEEG data, reading test and balance measures were additionally collected from a control group of 12 normally progressing children with no reading problems.

\section{Results}

The psychometric measures showed significant group differences (t-tests) in "with coloured lenses" and "without coloured lenses" condition at the .01 level or better, for all children. All children improved in one or other of the measures (working memory, speed of processing or literacy) either in terms of accuracy or speed, but some showed greater overall improvement. This correlated with the optometric measures of visual improvement in the "with coloured lenses" condition as opposed to the "without coloured lenses" condition. QEEG data showed the visual dyslexics had an abnormally high alpha peak in the without coloured lenses that attenuated when they were wearing their lenses. In other words, the "reading without coloured lenses" condition was significantly different to the "reading with coloured lenses" condition in the 9-11 Hz band over O1, O2, T5, T6, P3, P4, for these visual dyslexics.

\section{Conclusions}

These results suggest that prior to wearing coloured lenses their occipital-parietal-temporal areas were not active enough for adequate reading since their "without coloured lenses" measures resembled a 
normal eyes-closed alpha peak. Coloured lenses appear to enhance reading. Children who suffer from visually related perceptual problems (rather than auditory/phonological deficits) that inhibit reading are likely to benefit from coloured lenses. This is especially the case under fluorescent lighting. The implications for neurofeedback were discussed in terms of simultaneous activation of the occipital-parietal-temporal cortices.

\author{
Evaluating the Music of the Brain: Effects of Morphologic and \\ Functional Pathologies \\ M. Barry Sterman, PhD \\ School of Medicine, UCLA, Los Angeles, California \\ $<$ msterman@ucla.edu>
}

The new quantitative EEG metric termed "comodulation" is based on the correlation of trends in spectral magnitudes across time between electrode pairs in relevant frequency bands and functional states. As such it discloses the orchestration of neuronal regulation by revealing the underlying regional integration of neural networks. It has been shown that disturbances of comodulation in the unique dominant frequency of a given brain during the eyes-closed state can detect the frontal dysfunction of depression that has been documented by other brain imaging methods, as well as disturbed interactions between functional systems along the rostra-caudal axis of the cortex that are associated with somatic and behavioral issues. Further, this measure discloses disturbances in hemispheric interactions related to closed head injury. Focal lesions which corrupt local electrical activity are also identified with this metric. Further, its use clarifies some technical issues associated with EEG recording. The methodology, principles, and findings related to this new dimension of QEEG analysis were reviewed.

EEG Neurofeedback Treatment of Patients with Down Syndrome Tanju Surmeli, MD

Living Health Center, Istanbul, Turkey

$<$ neuropsychiatry@yahoo.com>

\title{
Introduction
}

Down syndrome is the most common identifiable cause of intellectual disability, accounting for almost one third of cases. It occurs 
equally in all races with an overall incidence of approximately one in 800 births. Neurofeedback (NF) is an operant conditioning method to increase or to decrease frequency bands of electroencephalography (EEG). An increasing number of clinicians use operant conditioning of EEG activity as a method of helping children with Attention Deficit Hyperactivity (ADD/ADHD) and Generalized Learning Disability (GLDO). Some of the Down syndrome children may have ADHD/ADD or GLDO, or both. We thought we might succeed applying neurofeedback to children with Down syndrome.

\section{Background}

Computer-assisted EEG neurofeedback treated subjects with Down syndrome. All eight subjects were medication-free during treatment. QEEG and Nxlink databases showed that almost all eight subjects have increased delta and theta waves in some area over the cortex and normal, increased or decreased beta waves over the cortex. Suggestions regarding diet were given to eight subjects and their parent's parenting skills were structured. All were not able to speak with more than one word in a sentence, with very limited vocabulary (between 5 and 10 words), usually pointed their finger to communicate, and were not able to carry basic comments, with very poor attention and concentration, and with poor memory, impulsivity and behavior problems. Some of them have balance problems, and they were unaware of their surroundings fully. The purpose of this study is to evaluate whether QEEG guided bipolar montage training is effective in developing speech, improving attention and concentration, improving learning, decreasing behavioral problems or impulsivity, and balance problems of Down Syndrome children.

\section{Methods}

Eight subjects with Down Syndrome were seen by the first author and by the special educator at baseline, 20th, 40th and 60th sessions. Before starting the neurofeedback exercises, the parents of the eight children with Down Syndrome (aged between 6 to 14) were given a questionnaire prepared by the center including questions about attention and concentration abilities, speech and language abilities, behavioral abilities and learning abilities. They had a QEEG recorded baseline and repeated at the end of the treatment. Neurofeedback trainings were performed by Lexicor Biolex software. NXLINK database was used to 
determine if they have ADHD/ADD/GLDO with clinical judgment of the first author. Neurofeedback therapy designed to normalize abnormal QEEG scores was provided to determine the effectiveness of this approach. Training continued until the subjects demonstrated improvement and, by self-reports of parents, indicated that significant improvement had occurred or until a total of 60 sessions were given. Lexicor QEEG signals were sampled at $128 \mathrm{~Hz}$. Neurofeedback trainings were performed by Lexicor Biolex software.

According to QEEG/NXLINK results F7-T5, F8-T6, P3-T5, P4-T6, CZ-C4, CZ-C3 electrode placement was used with a 0-4 Hz delta reward and inhibits at 4-8 Hz and CZ-C4 electrode placement with a 12-15 Hz SMR reward and inhibits at 4-8 Hz and F7-F5, F8-T6 electrode placement with a $15-18 \mathrm{~Hz}$ Beta reward, and inhibits at $4-8 \mathrm{~Hz}$.

\section{Results}

All seven children who received neurofeedback training showed significant improvement in their condition based on the questioner and parent interviewing. Mean age: $9.13 \mathrm{~N}=8, \mathrm{p}=0.000$ (or $\mathrm{p}<0.001$ ). One subject dropped the study after eight sessions.

All parents of the subjects reported that their children were able to speak four to five meaningful words in a sentence following the treatment, have a meaningful conversation with their parents and the others, their attention and concentration improved dramatically, their behavior became more mature and controllable, less impulsivity was seen, long and short term memory and their balance problems improved. Now they have a thinking brain and they can learn better and faster than before. Some of them have started going to normal school and their teacher reported that "their attention and memory is better than their normal peers."

Children with mosaic type genetic analyses thought to be showing ordinary development became not so different than their normal peers. The average age of death for a person with Down syndrome is in the mid-fifties. Changes with neurofeedback may increase in their life expectancy. Many have underestimated the abilities of these children and assumed that only certain achievements could be accomplished. Neurofeedback could change this view of teachers or special educators in the future. Further study with controls and additional outcome measures is warranted. 
Neurofeedback Treatment for Autistic Spectrum Disorders

Michael Thompson, MD and Lynda Thompson, PhD

Biofeedback Institute of Toronto, Canada

$<$ landmthompson@cs.com>

Autistic spectrum disorder clients have primary deficits in their ability to interpret social communications (innuendo, abstract meaning), appropriately initiate and maintain social interactions, handle anxiety, shift mental set, and sustain external attention span and response control. These deficiencies correspond to patterns observed on the electroencephalogram (EEG) that can include slowing at Pz, P4, T6, F4 and $\mathrm{PF} 1$ and differences in coherence and comodulation from normal databases. High theta activity at $\mathrm{Cz}$ is also observed in association with the problems with attention span. This presentation listed symptoms of PDD (Autism) and Asperger's syndrome, described typical cases, outlined assessment and intervention, and gave an overview of results of neurofeedback training with more than 60 cases, ages 5-51 including some with long-term follow-up.

The Effect of Distinct Neurofeedback Training Protocols on Working Memory, Mental Rotation and Attention Performance

David Vernon, PhD (1), Tobias Egner, PhD (2), Nick Cooper, MSc (3), Theresa Compton, BSc (3), Claire Neilands, BSc (3), Amna Sheri, BSc (3), and John Gruzelier, PhD (3)

(1) Department of Applied Social Sciences, Canterbury Christ Church University College, UK, (2) Functional Research Center, Columbia University, (3) Department of Cognitive Neuroscience \& Behaviour, Imperial College London, Charing Cross Hospital, London

$<$ d.j.vernon@cant.ac.uk>

\section{Background}

Based on research showing an association between theta $(4-7 \mathrm{~Hz})$ and working memory, alpha $(8-12 \mathrm{~Hz})$ and conceptual processing, and the sensorimotor rhythm (SMR) $(12-15 \mathrm{~Hz})$ and attention, we examined whether training individuals to enhance one of these frequency components would differentially influence their cognitive performance on a range of tasks.

\section{Methods}

Forty individuals were randomly allocated to one of four groups. Three completed a course of neurofeedback training enhancing a partic- 
ular component of their EEG (e.g., theta, alpha or SMR) while the fourth acted as a non-neurofeedback control group. The training consisted of eight sessions of neurofeedback over four weeks. Pre- and post-training all groups completed a range of tasks measuring working memory, image rotation and attention.

\title{
Results
}

Only those training the SMR rhythm exhibited changes in their SMR/ theta and SMR/beta EEG ratios. This resulted from a decrease in both theta and beta amplitudes and a concurrent increase in SMR amplitude. This group also showed a significant improvement in working memory performance and limited improvement in attentional performance.

\section{Conclusions}

Eight sessions of neurofeedback training is sufficient for healthy individuals to exhibit increased SMR activity. Enhanced SMR activity is associated with improved performance on a semantic working memory task and an attention task. These results were discussed in terms of different frequency components influencing distinct cognitive processes.

\author{
Attentional Set Shifting-Related EEG Alpha Power Desynchronization \\ in Sleep Apnea \\ Edwin Verstraeten, PhD and Raymond Cluydts, PhD \\ Department. of Cognitive and Physiological Psychology, Vrije Universiteit/ \\ Free University Brussels, Belgium \\ $<$ edwin.verstraeten@vub.ac.be>
}

\section{Background}

EEG slowing and often a prolonged P300 latency have been demonstrated in sleep apnea. These results were mostly interpreted as evidence of cerebral damage due to intermittent hypoxemia. However, as reduced alertness also produces EEG slowing or longer P300 latencies, it seems that alertness and cognitive processes were significantly confounded. 


\section{Methods}

Alpha power desynchronization was used because of its differential responsivity of $8-10 \mathrm{~Hz}$ and $10-12 \mathrm{~Hz}$ to non-specific alertness and taskrelated attention (e.g., Klimesch, Doppelmayr, Russegger, Pachinger, \& Schwaiger,1998; Sterman, 1999). Recently, we have shown that desynchronization of $8-10 \mathrm{~Hz}$ seemed to be related to phasic alertness; whereas, 10-12 Hz synchronization was associated with inaccurate attentional switching (Verstraeten \& Cluydts, 2002). Twenty sleep apnea patients and 24 age- and education-matched controls took part in this study. The EEG was recorded from AF3 during resting baseline and during attentional switching.

\section{Results}

Control subjects showed a decreasing trend in 8-10 Hz power during attentional switching, which was significantly different from patients who did not show any task-related alpha reactivity response.

\section{Conclusions}

Sleep apnea patients demonstrated a blunted phasic prefrontal activation during attentional switching. It is assumed that dampening of the diffuse thalamocortical projection system has caused a reduced level of phasic alertness that can explain the attenuation of prefrontal activation without the need to presume brain damage.

\section{REFERENCES}

Klimesch, W., Doppelmayr, M., Russegger, H., Pachinger, T., \& Schwaiger, J. (1998). Induced alpha band power changes in the human EEG and attention. Neuroscience Letters, 244, 73-76.

Sterman, M.B. (1999). Event-related EEG response correlates of task difficulty, sleep deprivation, and sensory distraction. In G. Pfurtscheller \& F. H. Lopes da Silva (Eds.), Event-related desynchronization. Handbook of electroencephalography and clinical neurophysiology: Revised Series (Vol. 6; pp. 233-242). Amsterdam: Elsevier Science.

Verstraeten, E. \& Cluydts, R. (2002). Attentional switching-related human EEG alpha oscillations. NeuroReport, 13, 681-684. 